\title{
Recovery and help system for the elders based on GSM
}

\author{
Zhiliang Chen ${ }^{\mathrm{a}}$, Liguo Tian ${ }^{\mathrm{b}}$, Meng Li \\ Key laboratory of Information Sensing \& Intelligent Control, \\ Tianjin University of Technology and Education, \\ Tianjin, China, 300222 \\ aczl_tj@163.com, btlg1234@163.com
}

\begin{abstract}
In modern society, along with the rapid development of society, the recovery and help system for the elders is born at the right moment. It discussed in detail the design idea and design scheme, and gave the working principle and the overall block diagram of the system based on the ATmega128 MCU and GSM module; secondly, provided the circuit diagram, the hardware structure to realize the function of the system and function of each hardware part; then discussed the system software design and the whole program. After tested, the system achieved the function of the whole system, basically meet the design requirements.
\end{abstract}

Keywords-ATmega128 MCU; GSM; GPS; LCD screen; voice prompt.

\section{INTRODUCTION}

With the development of high technology, people's life continuously covered by intelligent products. With the quickening pace of life, people's life concept is also changing; some of the products that are more close to people's life are becoming more and more popular. For the recovery and help system based on GSM, it is not only easy to remind some of things in the life, but also send text messages at emergency. Along with the social trend of population aging, digital community service has become an important international subject; under the government's attention, the related projects will be rapid promoted.

The paper used a combination of AVR SCM and GPS module effectively to design the recovery and help system. This system has the voice prompt, and tracking, sending information and other functions. The system uses the keyboard to set the voice prompt time and GPS to track the location and send a text message for help; besides, it uses ISD4004 voice module to record voice, at the same time, it also uses the LCD screen to display the time, set the time and user's geographical position.

\section{SYSTEM HARDWARE DESIGN}

\section{A. Overall design}

The design uses ATmega128 microcontroller as the core of detection and control. It uses the key to set the time, using TTL serial port to control ISD4004 voice chip to realize voice prompt, and it uses the PC to communicate with SCM through the serial port, so as to debug for the system with $4 * 4$ keyboards. At this point, the system Maximize the use of all the resources of AVR SCM.
In order to meet the design requirements, the system should be composed of the following 7 parts: MCU part, the keyboard part, the power part, the display module, GSM module and GPS module.

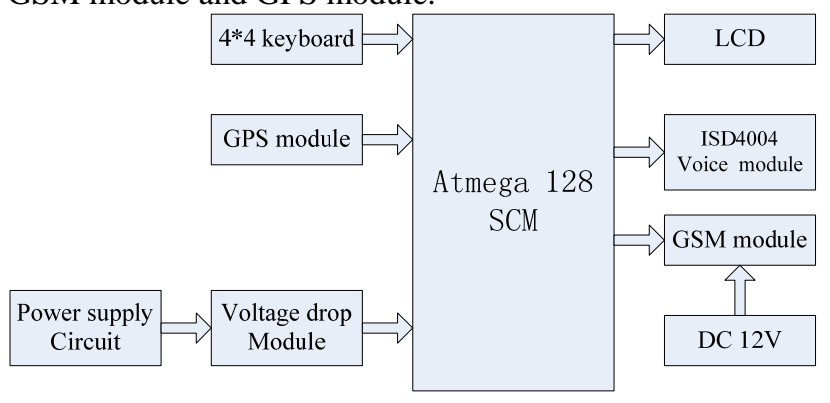

Fig.1. System schematic diagram

Function of each module:

SCM: be responsible for the collection of information of the keyboard and GPS, control the liquid crystal display screen; control the GSM module to send text messages.

Matrix keyboard part: man-machine interface input part. It can send the help information by separate keyboard, set the target mobile phone number through software, and test the model through the keyboard switch menu.

Power supply: the external power supply interface and a voltage stabilizing filter, to provide a stable power supply system.

The displayed part: man-machine interface output part, display various data and state of the system.

GSM module: sending short message through mobile wireless networks and through satellite positioning calculate user's longitude and latitude and send them to the microprocessor.

\section{B. Module design}

\section{1) SCM module}

The system uses ATmega128 microcontroller as the minimum system of central processor, control the voice chip by TTL communication mode, detect the state of key to control voice playing etc... ATmega128 microprocessor is an 8-bit AVR microprocessor with high performance, low power consumption, the use of advanced RISC structure, the integration of the JTAG interface, and can be achieved on the Flash, EEPROM, fuse and lock bits of programming through JTAG interface, improving the 
efficiency of debugging. The minimum system is shown in figure 2 .

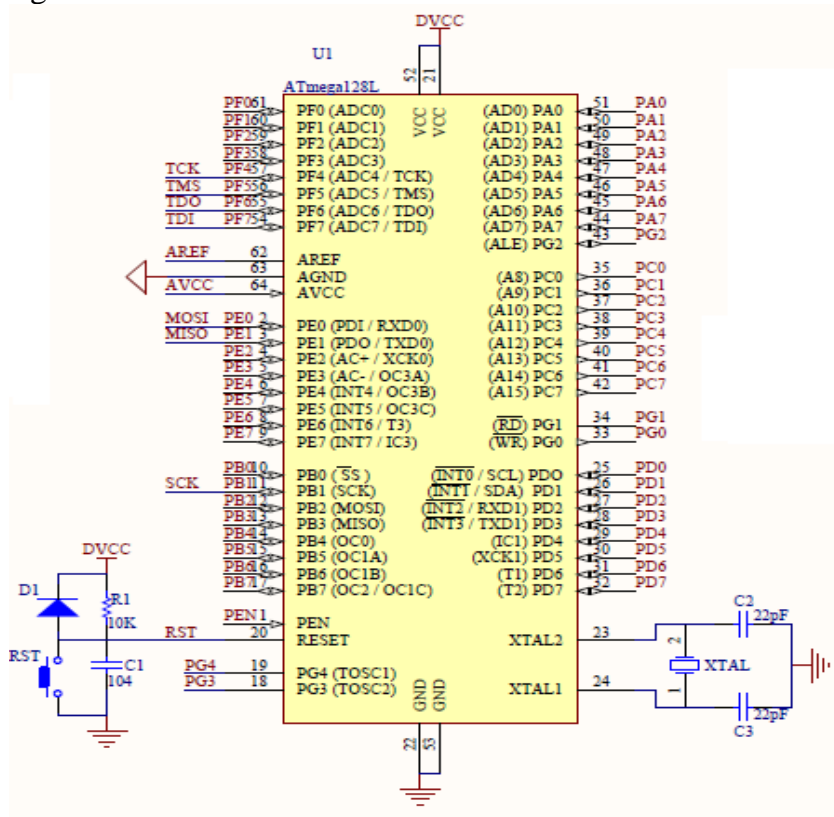

Fig.2. ATmega128 SCM minimum system

\section{2) GPS module}

GPS (Global Positioning System) is the abbreviation of global positioning system, which can provide all-weather positioning, time service and velocity measurement.

The system used Siemens Industrial GSM module TC35i, which can realize the short message service quickly and safely (Short Message Service). TC35i module mainly consists of a GSM baseband processor, GSM RF module, power supply module, flash memory, ZIF connector and antenna interface. Its compact design greatly reduces the volume of the product, and it is compatible with GSM2/2+, conforms to ETSI standard GSM0707 and GSM0705, the working voltage of module is $3.3 \sim 4.8 \mathrm{~V}$, compatible with dual frequency (GSM 900/GSM L800), has RS232 data communication port. The module integrates RF circuit and baseband module, provides the user with the AT command interface and fast, reliable, secure transmission for data, voice and short message. TC35i has 40 pin that lead by ZIF. The external wiring diagram as follows:

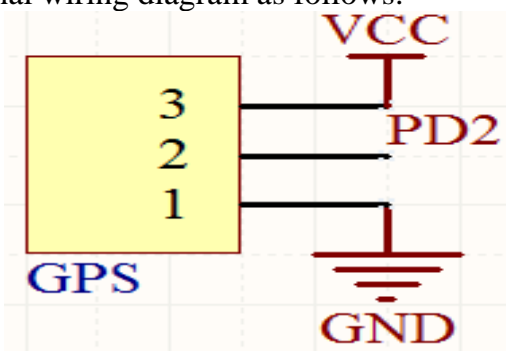

Fig.3. Connection diagram between ATmega128 and GPS

\section{3) Voice module}

The speech function of the system is realized through ISD4004 voice chip, the pin diagram as shown in figure 4 . Working voltage of ISD4004 series is 3.3V; the recording time is 8 minutes or 16 minutes, sound quality is well, suitable for mobile phones and other portable electronic products.

\section{4) Keyboard module}

This system due to the different input timing and independent keys, so the needed key number is more, in order to reduce the system resources of $\mathrm{I} / \mathrm{O}$, the design of the system function keyboard is $4 * 4$ matrix keyboard, the keyboard matrix is composed of the row and column lines, and the keys are at the intersections of the row and column lines for connection number keys, a keyboard matrix containing the keyboard is equal to the number of rows and columns, the realization of the 16 key input only need $8 \mathrm{I} / \mathrm{O}$ port, so that greatly reduces the system resource. $4 * 4$ matrix keyboard interface circuit, as shown in figure 5 .

\section{SYSTEM SOFTWARE DESIGN}

The elderly recovery help system based on GSM uses the modular program method, including the main program, key scanning program, GSM messages sent procedures, GPS positioning program and voice broadcast procedures.

The main program is mainly to complete the initialization of the system, calling all function programs, and transmission of each function program. But the main program is so less as to write it and positioning program of GPS module together.

Keyboard scanning procedures, due to different time setting, so need more, using the keyboard matrix, which can meet the requirements of the system and can save the $\mathrm{I} / \mathrm{O}$ resource.

Based on the key state determined by SCM, GSM SMS program will execute the sending command; the voice prompt will be available after the message is sent successfully.

Voice record-replay procedure mainly completes voice recording, and broadcast content is recorded before, also can be modify by this procedure.

\section{A. Main program}

The main program of the system can realizes the initialization of the system, the calling of service program, and the detection and treatment of the flag bit established for interruption, and monitoring the process, so as to complete various functions.

The flow chart of main program is shown in figure 6 . 


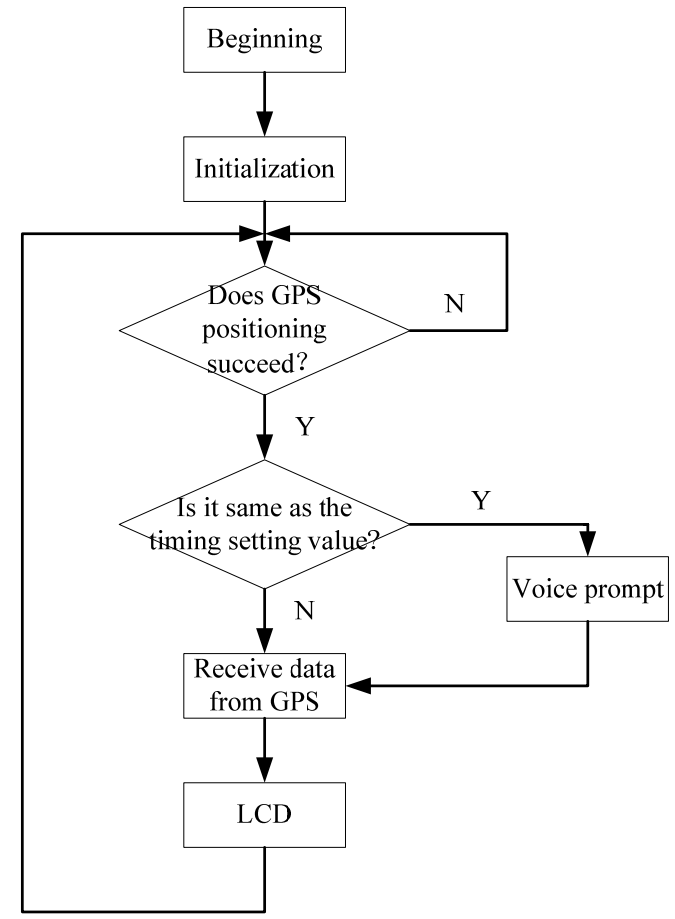

Fig.6. Flow chart of the main program

\section{B. SMS procedure of GSM module}

The GSM module of the system only uses the function of sending information. It is mainly determined by the key state which is received by the keyboard to let the GSM module performs send SMS, the module flow chart, as shown in figure 7.

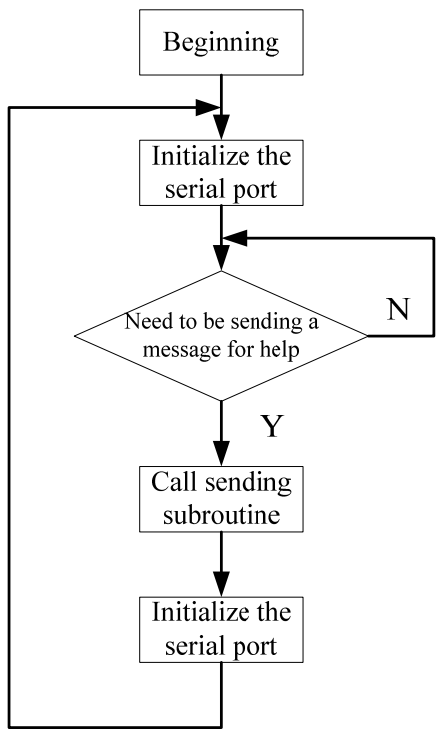

Fig.7. The flow chart of GSM module

\section{SYSTEM DEBUGGING}

The system debugging mainly includes two aspects: software debugging and hardware debugging. System hardware debugging mainly includes the system power supply debugging, ATmega128 MCU minimum system debugging and audio amplifier circuit debugging so on.

\section{CONCLUSIONS}

The paper at first designed the overall block diagram of the system, and then introduced each hardware module design; at last it introduced the design method of system software. The system adopted the modular programming method; the software includes the main program, GSM program, keyboard scanning procedures and voice recording procedures. After debugging, the system basically meets the design requirements, can be used in the practical life.

\section{ACKNOWLEDGMENT}

This work was supported by China'863'High-Tech Program under Grant No. SS2013AA03120.

\section{CORRESPONDING AUTHOR}

Name: Zhiliang CHEN,

Email:czl_tj@163.com,

Mobile phone: +86-13820623957

\section{REFERENCES}

[1] Honggang Fan, Yanyou Song. AVR SCM self-study notes. Beijing: Beijing University of Aeronautics and Astronautics Press, 2012

[2] Jianqing Liu. Easy to study AVR C language. Beijing: Beijing University of Aeronautics and Astronautics Press, 2011

[3] Jun Zhang. Development of typical examples of AVR SCM application system. Beijing: China Electric Power Press, 2005

[4] Chao Ma. Principle and application of advanced 8-bit SCM ATmega128 guide. Beijing: Beijing University of Aeronautics and Astronautics Press, 2004

[5] Yuqing $\mathrm{Hu}$, Zhaojun Shu. Design of electronic recording system based on ISD4004. Journal of Beijing College of electric power, 2009, 4:157-158.

[6] Xiaodong Zhang. Design of intelligent voice prompt system based on ISD4004. Journal of West Anhui University, 2008, 24:69-71.

[7] Wei Zhang. GSM Network Optimization -- the principle and engineering (Second Edition). The posts and Telecommunications Press, 2010 


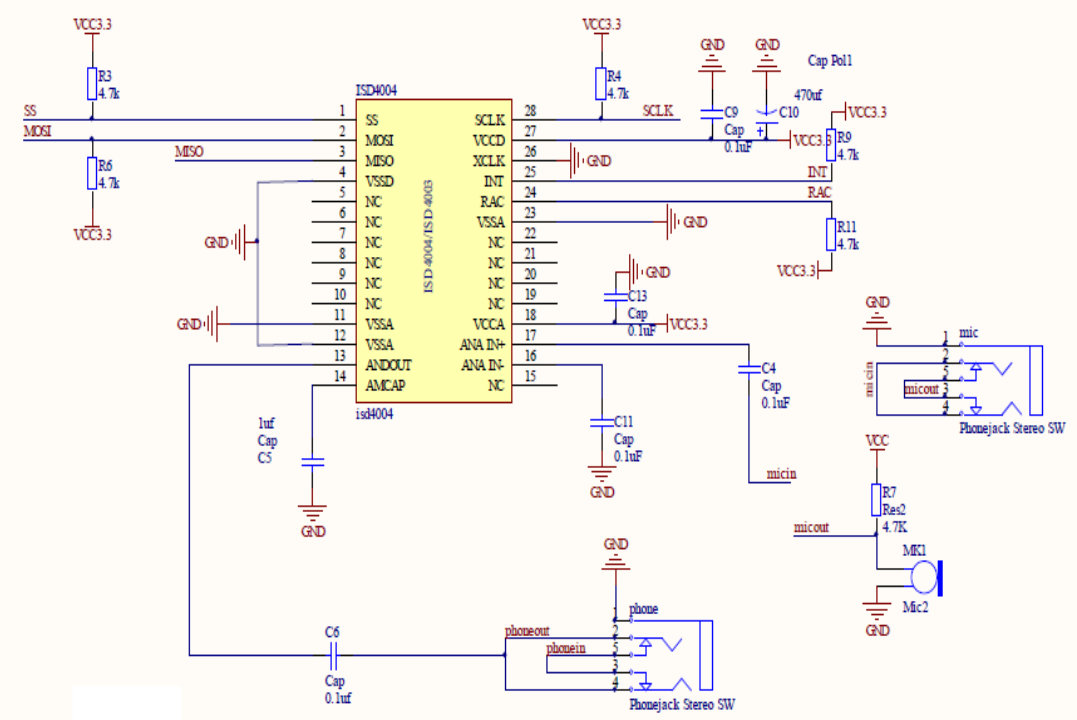

Fig.4. ISD4004 principle diagram

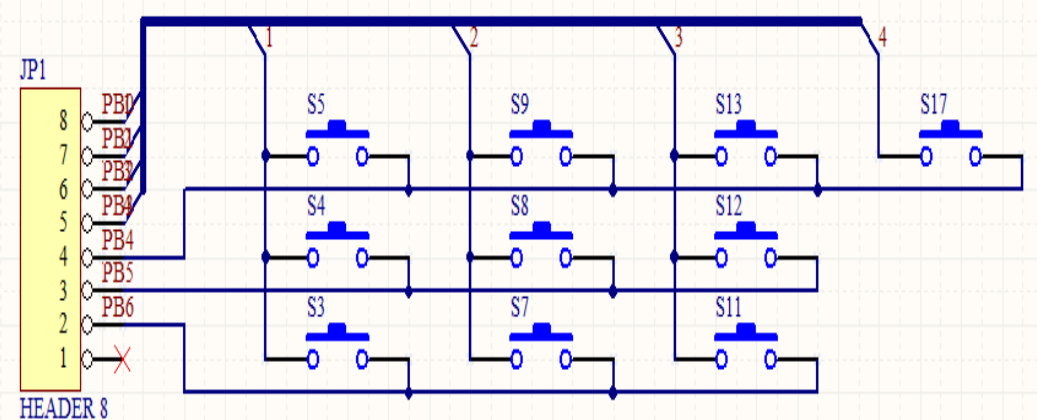

Fig.5. 4*4 matrix keyboard interface circuit 